\title{
Synthesis of substituted 1, 2, 4-triazole derivatives by Microwave irradiation
}

\author{
Ashish Asrondkar ${ }^{1}$, Vrushali Patil ${ }^{1}$, Shantanu Pande ${ }^{1}$, A.S.Bobade ${ }^{1}$, \\ A.S.Chowdhary ${ }^{1}$ \\ ${ }^{I}$ Department of Chemotherapy, Haffkine Institute for Training, Research and Testing, India
}

\begin{abstract}
Various substituted Triazole-Thiol containing different functional group have been synthesized by microwave method. The title product 1-[(3H-indol-2-ylamino) methyl]-4-phenyl-4, 5-dihydro-1H-1, 2, 4triazole-3-thiol is synthesized by using amino benzothiazole. The final structures have been established on the basis of their chemical analysis and spectral data. All micro-wave synthesized compounds results into good yield as compared to conventional method of which fluoro substituted compound shows maximum yield.
\end{abstract}

Keyword: Benzothiazole, 1,2,4-triazole,Microwave irradiation etc

\section{INTRODUCTION}

1,2,4-Triazoles and their derivatives are important group of heterocyclic compounds characterized by a five-membered ring of two carbons and three nitrogen atoms. The biological activity of 1,2,4-triazoles and their derivatives have been demonstrated by various studies ${ }^{1,2}$. For instance, data came from previous investigations showed that 1,2,4-triazole nucleus posses a wide range of pharmacological activities such as analgesic ${ }^{3}$, antibacterial ${ }^{4}$, antifungal ${ }^{5}$, anti-inflammatory ${ }^{6}$

Microwave assisted organic synthesis ${ }^{7}$ became an increasingly popular technique in academic and industrial research laboratories, due to certain advantages, particularly shorter reaction times and rapid optimization of chemical reactions. The experimental technique applied for the organic syntheses described below, is based on microwave power processing of materials using a dynamic control of the microwave power magnetron. The aim of this work was to test the efficiency of this new dynamic microwave power system in the organic synthesis by optimizing the chemical synthesis of some interesting 1,2, 4-triazoles derivatives.

\section{MATERIALS AND MethodS}

All raw materials used in the synthesis have been obtained from M/S Fluka AG (Bachs, Switzerland) and M/S Sigma-Aldrich chemicals and Co. Inc. (Milwoukee, WI,USA). Melting points were recorded on a Thermonik Melting point Apparatus (Campbell Electronics, Mumbai, India) and are uncorrected. IR spectra were recorded on a IR-Affinity, Shimadzu using DRS system. ${ }^{1} \mathrm{H}-\mathrm{NMR}$ spectra have been recorded on a JEOL AL-300 FT-NMR spectrometer (300 MHz, JEOL Ltd., Tokyo, Japan), using TMS as internal standard in solvent DMSO. Elemental analysis has been carried out on a C, H, and N Elemental Analyzer (Thermo-Finnigan Flash EA 1112, Italy).

\subsection{EXPERIMENTAL}

\subsubsection{Preparation of ethyl (1,3-benzothiazol-2-ylamino) aCetate (COMPOUND 2)}

Dissolve the 1,3-benzothiazol-2-amine $(2.0 \mathrm{~mol})$ in acetone and ethyl chloroacetate $(2.0 \mathrm{~mol})$ was added drop-wise in presence of $\mathrm{k}_{2} \mathrm{CO}_{3}(8 \mathrm{~g})$ in the mixture with stirring. the reaction mixture was refluxed for 20 hours and the product was isolated. the completion of the reaction was mentioned by TLC. Yield 74\%; white colour solid; mp; $133^{0} \mathrm{C}^{1} \mathrm{H}$ NMR(400 MHz, DMSO-d6) d (ppm) $10.0(\mathrm{~s}, 1 \mathrm{H}), \quad 4.13-4.24 \quad(\mathrm{~s}, \quad 2 \mathrm{H})$ Anal. calcd for $\mathrm{C}_{2} \mathrm{H}_{3} \mathrm{NO}_{2}: \mathrm{C}, 30.76 ; \mathrm{H}, 2.565 ; \mathrm{N} 11.36$ Found: C, 30.37; H, 2.16; N, 12.03 . IR (KBr) $\mathrm{cm}^{-1}$ : 3415(-NH), 2943(-CH3), 1719(C=O),MS (m/z): $150\left[\mathrm{M}^{+}\right]\left(\mathrm{C}_{7} \mathrm{H}_{6} \mathrm{~N}_{2} \mathrm{~S}^{+}\right), 134 \quad\left(\mathrm{C}_{7} \mathrm{H}_{4} \mathrm{NS}\right)$

\subsubsection{Preparation OF 2-(1,3-BENZOThiaZOl-2-YLAMino) aCETOHYDRAZIDE} (COMPOUND 3)[BY CONVENTIONAL METHOD]

Compound 2(1 mol) dissolve in ethanol treated with hydrazine hydrate $(1 \mathrm{~mol})$ was refluxed for $6 \mathrm{hrs}$. The reaction was cooled, poured into ice cold water. Solid product was filtered, dried and Recrystallized from ethanol.

\section{[By Microwave Irradiation method]}

A mixture of compound 1, hydrazine hydrate and ethanol was placed in microwave oven and irradiated for 6 min. Completion of the reaction was monitored by TLC, mixture was cooled and the resulting solid was filtered and recrystallized from ethanol. 
Synthesis of substituted 1, 2, 4-triazole derivatives by Microwave irradiation.

Yield $62 \%$; white colour solid; $\mathrm{mp} ; 188^{\circ} \mathrm{C}$

${ }^{1} \mathrm{H}$ NMR(400 MHz, DMSO-d6) d (ppm) 6.98-7.65 (m, 5H Ar-H), 10.35 (s, 1H) Anal. calcd for $\mathrm{C}_{10} \mathrm{H}_{6} \mathrm{O}_{3}: \mathrm{C}$, 68.96; H, 3..44; Found: C, 67.37; H, 2.16

IR $(\mathrm{KBr}) \mathrm{cm}^{-1}:$ 1645(C-O-C), 2953(-CH3), 1720(C=O)

MS (m/z): $236\left[\mathrm{M}^{+}\right]\left(\mathrm{C}_{11} \mathrm{H}_{12} \mathrm{~N}_{2} \mathrm{O}_{2} \mathrm{~S}^{+}\right), 207\left(\mathrm{C}_{9} \mathrm{H}_{7} \mathrm{~N}_{2} \mathrm{O}_{2} \mathrm{~S}\right) 163\left(\mathrm{C}_{8} \mathrm{H}_{7} \mathrm{~N}_{2} \mathrm{~S}\right), 149\left(\mathrm{C}_{7} \mathrm{H}_{5} \mathrm{~N}_{2} \mathrm{~S}\right), 134\left(\mathrm{C}_{7} \mathrm{H}_{4} \mathrm{NS}\right)$

2.1.3 PREPARation OF 4-[(1, 3-BENZOTHIAZOL-2-Ylamino) METHYL]-4, 5-DIHYDRO-1, 3, 4-OXADiAZOLE-2THIOL (COMPOUND 4)

Salt formation of compound $2(1 \mathrm{~mol})$ was prepared by using by alcohol in presence of KOH $(1 \mathrm{~mol}) \quad$ and refluxed for $1 \mathrm{hr}$ on water bath. The solution of carbon disulphide in alcohol was added drop wise. Reaction mixture was refluxed till evolution of $\mathrm{H}_{2} \mathrm{~S}$ gas ceases. Resulted product recrystallized from ethanol.

Yield $69 \%$; white colour solid; $\mathrm{mp} ; 216^{\circ} \mathrm{C}$

${ }^{1} \mathrm{H}$ NMR(400 MHz, DMSO-d6) d (ppm) 6.45-7.65 (m, 5H Ar-H), 10.35 (s, 1H),2.34 (s, 3H) Anal. calcd for $\mathrm{C}_{11} \mathrm{H}_{8} \mathrm{O}_{3}: \mathrm{C}, 70.25 ; \mathrm{H}, 4.25$; Found: $\mathrm{C}, 69.37 ; \mathrm{H}, 4.16$

IR $(\mathrm{KBr}) \mathrm{cm}^{-1}: 1615(\mathrm{C}-\mathrm{O}-\mathrm{C}), 2943(-\mathrm{CH} 3), 1745(\mathrm{C}=\mathrm{O})$

MS (m/z): $266\left[\mathrm{M}^{+}\right]\left(\mathrm{C}_{10} \mathrm{H}_{10} \mathrm{~N}_{4} \mathrm{OS}_{2}^{+}\right), 233\left(\mathrm{C}_{10} \mathrm{H}_{9} \mathrm{~N}_{4} \mathrm{OS}\right), 163\left(\mathrm{C}_{8} \mathrm{H}_{7} \mathrm{~N}_{2} \mathrm{~S}\right), 149\left(\mathrm{C}_{7} \mathrm{H}_{5} \mathrm{~N}_{2} \mathrm{~S}\right), 134 \quad\left(\mathrm{C}_{7} \mathrm{H}_{4} \mathrm{NS}\right)$.

2.1.4 Preparation of 1-[(3H-indol-2-ylamino) methyl]-4-phenyl-4, 5-dihydro-1H-1, 2, 4-triazole-3-thiol (Compound 5a-f)

The mixture of compound $3 \&$ substituted aniline was dissolve in ethanol. The resulted mix was refluxed for 8 hrs. Final product was recrystallized from ethanol.

2.1.4.1 Preparation of 1-[(3H-indol-2-ylamino) methyl]-4-phenyl-4, 5-dihydro- $1 H$-1, 2, 4-triazole-

3thiol _(5a)

${ }^{1} \mathrm{H}$ NMR(400 MHz, DMSO-d6) d (ppm) 6.45-7.65 (m, 9H Ar-H), 10.20 (s, 2H),5.34 (s, 1H), 5.21 ( s,

Anal. calcd for $\mathrm{C}_{16} \mathrm{H}_{15} \mathrm{~N}_{5} \mathrm{~S}_{2}: \mathrm{C}, 56.30 ; \mathrm{H}, 4.03$, N, 20.52; Found: C, 56.37; H, 4.16. N, 19.82

IR (KBr) cm ${ }^{-1}$ 2943(-CH3), 3103(-OH).

$\operatorname{MS}(\mathrm{m} / \mathrm{z}): 341\left[\mathrm{M}^{+}\right]\left(\mathrm{C}_{16} \mathrm{H}_{15} \mathrm{~N}_{5} \mathrm{~S}_{2}^{+}\right), 264\left(\mathrm{C}_{10} \mathrm{H}_{10} \mathrm{~N}_{5} \mathrm{~S}_{2}\right), 231\left(\mathrm{C}_{10} \mathrm{H}_{9} \mathrm{~N}_{5} \mathrm{~S}\right), 163\left(\mathrm{C}_{8} \mathrm{H}_{7} \mathrm{~N}_{2} \mathrm{~S}\right), 149 \quad\left(\mathrm{C}_{7} \mathrm{H}_{5} \mathrm{~N}_{2} \mathrm{~S}\right)$,

$134\left(\mathrm{C}_{7} \mathrm{H}_{4} \mathrm{NS}\right)$

2.1.4.3Preparation of 4-\{1-[(1,3-benzothiazol-2-ylamino)methyl]-3-sulfanyl-1,5-dihydro-4H-1,2,4-triazol4-yl\}phenol (5b)

${ }^{1} \mathrm{H}$ NMR(400 MHz, DMSO-d6) d (ppm) 6.45-7.65 (m, 9H Ar-H), 11.20 (s, 1H)) Anal. calcd for $\mathrm{C}_{16} \mathrm{H}_{15} \mathrm{~N}_{5} \mathrm{OS}_{2}$ :C, 53.78; H, 4.48, N, 19.60; Found: C, 53.72; H, 4.16, N, 19.79

IR (KBr) cm $\mathrm{cm}^{-1}: 2943(-\mathrm{CH} 3), 3103(-\mathrm{OH})$.

$\mathrm{MS}(\mathrm{m} / \mathrm{z}):): 357\left[\mathrm{M}^{+}\right]\left(\mathrm{C}_{16} \mathrm{H}_{15} \mathrm{~N}_{5} \mathrm{OS}_{2}^{+}\right), 340\left(\mathrm{C}_{16} \mathrm{H}_{14} \mathrm{~N}_{5} \mathrm{~S}_{2)}, 264\left(\mathrm{C}_{10} \mathrm{H}_{10} \mathrm{~N}_{5} \mathrm{~S}_{2}\right), 231\left(\mathrm{C}_{10} \mathrm{H}_{9} \mathrm{~N}_{5} \mathrm{~S}\right), 163\left(\mathrm{C}_{8} \mathrm{H}_{7} \mathrm{~N}_{2} \mathrm{~S}\right)\right.$, $149\left(\mathrm{C}_{7} \mathrm{H}_{5} \mathrm{~N}_{2} \mathrm{~S}\right), 134\left(\mathrm{C}_{7} \mathrm{H}_{4} \mathrm{NS}\right)$.

2.1.4.4Preparation of 1-[(1,3-benzothiazol-2-ylamino)methyl]-4-(4-fluorophenyl)-4,5-dihydro-1H-1,2,4triazole-3-thiol $(5 c)$

${ }^{1} \mathrm{H}$ NMR(400 MHz, DMSO-d6) d (ppm) 6.95-7.89 (m, 9H Ar-H), 11.20 (s, 1H), 2.43 (s, 2H) Anal. calcd for $\mathrm{C}_{16} \mathrm{H}_{14} \mathrm{~N}_{5} \mathrm{~S}_{2} \mathrm{~F}: \mathrm{C}, 53.63 ; \mathrm{H}, 3.91$, N, 19.55; Found: C, 55.37; H, 3.16. N, 18.32

IR $(\mathrm{KBr}) \mathrm{cm}^{-1}$ : $3235(-\mathrm{NH}), 1896(\mathrm{C}=\mathrm{C})$

MS (m/z): ): $358\left[\mathrm{M}^{+}\right]\left(\mathrm{C}_{16} \mathrm{H}_{14} \mathrm{~N}_{5} \mathrm{~S}_{2} \mathrm{~F}^{+}\right), 340\left(\mathrm{C}_{16} \mathrm{H}_{14} \mathrm{~N}_{5} \mathrm{~S}_{2}\right), 264\left(\mathrm{C}_{10} \mathrm{H}_{10} \mathrm{~N}_{5} \mathrm{~S}_{2}\right), 231\left(\mathrm{C}_{10} \mathrm{H}_{9} \mathrm{~N}_{5} \mathrm{~S}\right), 163\left(\mathrm{C}_{8} \mathrm{H}_{7} \mathrm{~N}_{2} \mathrm{~S}\right)$, $149\left(\mathrm{C}_{7} \mathrm{H}_{5} \mathrm{~N}_{2} \mathrm{~S}\right), 134\left(\mathrm{C}_{7} \mathrm{H}_{4} \mathrm{NS}\right)$.

2.1.4.5 Preparation of 1-[(1,3-benzothiazol-2-ylamino)methyl]-4-(4-methoxyphenyl)-4,5-dihydro-

$1 H-$ 1,2,4-triazole-3-thiol (5d)

${ }^{1} \mathrm{H}$ NMR(400 MHz, DMSO-d6) d (ppm) 6.45-7.65 (m, 9H Ar-H), 11.20 (s, 1H).2.45 ( s, 2H) Anal. calcd for $\mathrm{C}_{17} \mathrm{H}_{17} \mathrm{~N}_{5} \mathrm{OS}_{2}: \mathrm{C}, 54.49 ; \mathrm{H}, 4.58, \mathrm{~N}, 18.86$; Found: C, 54.07; H, 4.98. N, 18.12

IR (KBr) cm $\mathrm{cm}^{-1}$ : 1675(C-O-C), 2943(-CH3).

MS (m/z): ): $371\left[\mathrm{M}^{+}\right]\left(\mathrm{C}_{17} \mathrm{H}_{17} \mathrm{~N}_{5} \mathrm{OS}_{2}^{+}\right), 340\left(\mathrm{C}_{16} \mathrm{H}_{14} \mathrm{~N}_{5} \mathrm{~S}_{2)}, 264\left(\mathrm{C}_{10} \mathrm{H}_{10} \mathrm{~N}_{5} \mathrm{~S}_{2}\right), 231\left(\mathrm{C}_{10} \mathrm{H}_{9} \mathrm{~N}_{5} \mathrm{~S}\right), 163\left(\mathrm{C}_{8} \mathrm{H}_{7} \mathrm{~N}_{2} \mathrm{~S}\right)\right.$, $149\left(\mathrm{C}_{7} \mathrm{H}_{5} \mathrm{~N}_{2} \mathrm{~S}\right), 134\left(\mathrm{C}_{7} \mathrm{H}_{4} \mathrm{NS}\right)$.

2.1.4.6Preparation of 1-[(1,3-benzothiazol-2-ylamino)methyl]-4-(3,4-dichlorophenyl)-4,5-dihydro-

$1 H-$ 1,2,4-triazole-3-thiol (5e)

${ }^{1} \mathrm{H}$ NMR(400 MHz, DMSO-d6) d (ppm) 6.45-7.65 (m, 9H Ar-H), 11.20 (s, 1H) Anal. calcd for $\mathrm{C}_{16} \mathrm{H}_{14} \mathrm{~N}_{5} \mathrm{~S}_{2} \mathrm{Cl}_{2}$ :C, 46.82; H, 3.41, N, 17.07; Found: C, 46.01; H, 3.16, N, 18.00 .

IR $(\mathrm{KBr}) \mathrm{cm}^{-1}$ : 2943(-CH3), 785(C-Cl). 
$\operatorname{MS}(\mathrm{m} / \mathrm{z}): 410\left[\mathrm{M}^{+}\right]\left(\mathrm{C}_{16} \mathrm{H}_{14} \mathrm{~N}_{5} \mathrm{~S}_{2} \mathrm{Cl}_{2}^{+}\right), 340\left(\mathrm{C}_{16} \mathrm{H}_{14} \mathrm{~N}_{5} \mathrm{~S}_{2}\right), 263\left(\mathrm{C}_{10} \mathrm{H}_{9} \mathrm{~N}_{5} \mathrm{~S}_{2}\right), 230\left(\mathrm{C}_{10} \mathrm{H}_{8} \mathrm{~N}_{5} \mathrm{~S}\right)$.

2.1.4.7Preparation of 1-[(1,3-benzothiazol-2-ylamino)methyl]-4-( 4-methylphenyl)-4,5-dihydro-

$1 H-$ 1,2,4-triazole-3-thiol (5f)

${ }^{1} \mathrm{H}$ NMR(400 MHz, DMSO-d6) d (ppm) 6.45-7.65 (m, 9H Ar-H), 11.20 (s, 1H) Anal. calcd for $\quad \mathrm{C}_{17} \mathrm{H}_{17} \mathrm{~N}_{5} \mathrm{~S}_{2}$ :C, 57.46; H, 4.78, N, 19.71; Found: C, 57.79, H, 4.89,N,19.24

IR $(\mathrm{KBr}) \mathrm{cm}^{-1}: 3254(-\mathrm{NH}), 1254(\mathrm{C}-\mathrm{H})$

MS (m/z): $355\left[\mathrm{M}^{+}\right]\left(\mathrm{C}_{17} \mathrm{H}_{17} \mathrm{~N}_{5} \mathrm{~S}_{2}{ }^{+}\right), 340\left(\mathrm{C}_{16} \mathrm{H}_{14} \mathrm{~N}_{5} \mathrm{~S}_{2}\right)$,

\section{Table 1: Schematic Representation of Titled Compounds}

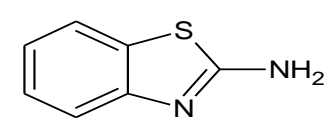

1,3-benzothiazol-2-amine 1

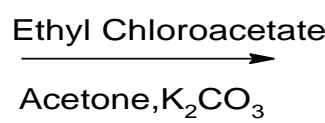

Acetone, $\mathrm{K}_{2} \mathrm{CO}_{3}$

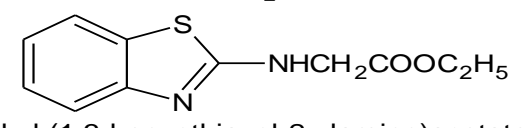

ethyl (1,3-benzothiazol-2-ylamino)acetate 2

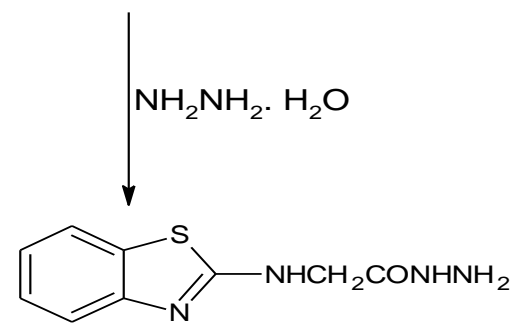

2-(1,3-benzothiazol-2-ylamino)acetohydrazide

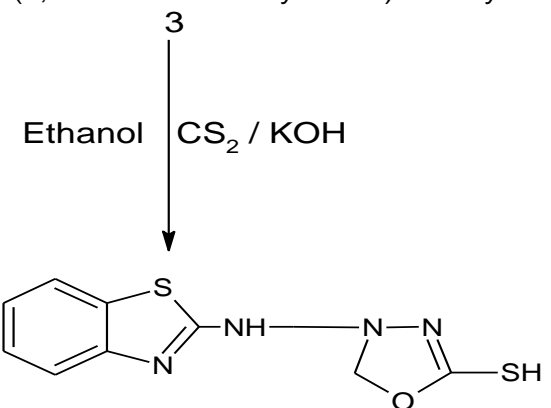

4-[(1,3-benzothiazol-2-ylamino)methyl]-4,5-dihydro-1,3,4-oxadiazole-2-thiol $\left.\right|^{4} \mathrm{R}-\mathrm{NH}_{2}$

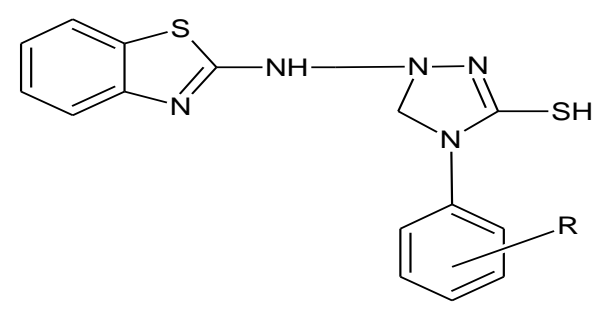

1-[(1,3-benzothiazol-2-ylamino)methyl]-4-phenyl-4,5-dihydro-1 H-1,2,4-triazole-3-thiol 
Synthesis of substituted 1, 2, 4-triazole derivatives by Microwave irradiation.

\subsection{Table 2: Comparative table between Conventional Method \& Microwave} Irradiation Method.

\begin{tabular}{|c|c|c|c|c|c|c|c|}
\hline \multirow{2}{*}{ Sr. No. } & \multirow{2}{*}{ Comp. } & \multirow{2}{*}{$\mathrm{R}$} & \multirow{2}{*}{$\mathrm{MP}\left({ }^{0} \mathrm{C}\right)$} & \multicolumn{2}{|c|}{ Time } & \multicolumn{2}{|c|}{ Yield \% } \\
\hline & & & & Conventional & MW & Conventional & MW \\
\hline 1 & $5 a$ & $\mathrm{H}$ & 188 & $8 \mathrm{hrs}$ & $4 \mathrm{~min}$ & $54 \%$ & $71 \%$ \\
\hline 2 & $5 b$ & $\mathrm{OH}$ & 197 & $7 \mathrm{hrs}$ & $6 \mathrm{~min}$ & $59 \%$ & $73 \%$ \\
\hline 3 & $5 c$ & $\mathrm{~F}$ & 205 & $8 \mathrm{hrs}$ & $5 \mathrm{~min}$ & $63 \%$ & $80 \%$ \\
\hline 4 & $5 d$ & $\mathrm{OCH}_{3}$ & 179 & $6 \mathrm{hrs}$ & $5 \mathrm{~min}$ & $44 \%$ & $62 \%$ \\
\hline 5 & $5 \mathrm{e}$ & $\mathrm{Di}-\mathrm{Cl}$ & 165 & $6 \mathrm{hrs}$ & $6 \mathrm{~min}$ & $50 \%$ & $65 \%$ \\
\hline 6 & $5 f$ & $\mathrm{CH}_{3}$ & 218 & $5 \mathrm{hrs}$ & $5 \mathrm{~min}$ & $56 \%$ & $75 \%$ \\
\hline
\end{tabular}

\section{RESULTS}

As the graphic enhancements in the speed of reactions and in yields shown by the microwave assisted methods compared to conventional methods are striking, undoubtedly, microwaves are going to be highly important in future synthesis of heterocycles. Also heterocyclic compounds being the most biologically active and are highly important in combinatorial chemistry to identify leads and to optimize structures, the applications of microwaves will only increase in future.

\section{CONCLUSION}

In the present research work serious of various substituted triazoles derivatives containing 1-[( $3 H$ indol-2-ylamino) methyl]-4-phenyl-4, 5-dihydro-1H-1, 2, 4-triazole-3-thiol were synthesized as mentioned in the scheme and experimental work. Compounds were synthesized by microwave as well as with conventional method and only time factor and percentage yield were compared. All these compounds were tested for their purity by TLC and melting point. The structures were confirmed by IR, NMR and CHN analysis. The microwave assisted organic synthesis required less time and also percentage yield was more compared with conventional method.

\section{ACKNOWLEDGEMENTS}

The authors are thankful to SAIF, IIT, Powai, Mumbai for carrying out the elemental analysis (CHN) and also thankful to SAIF, Patiala University, Punjab for recording the NMR spectra.

\section{REFERENCES}

[1]. Y.A. Al-Soud, H.A. Mohammed and A. Abo-Amer, Microwave-assisted synthesis and antioxidant properties of some new 1,2,4triazole derivatives, Jordan Journal of Chemistry, 5 2010, 119-129

[2]. R. Agrwal and S.S. Pancholi, Synthesis, characterization and evaluation of antimicrobial activity of a series of 1,2,4-triazoles, Der Pharma Chemica, 3 2011, 32-40.

[3]. A. Cansiz, S. Servi, M. Koparir, M. Altintas and M. Digrak. Synthesis and biological activity of Mannich bases of 5-(2-furyl)-1,2,4triazole-3-thiones, Journal of the Chemical Society of Pakistan, 23,2001, 237-240.

[4]. S. Jantová, G. Greif, R. Pavlovičová and L'. Čipák, Antibacterial effects of some 1-substituted 1,2,4-triazoles, Folia Microbiologica, 43,1998, 75-78

[5]. Z. Rezaeia, S. Khabnadideha, K. Pakshirb, Z. Hossainia, F. Amiria and E. Assadpour, Design, synthesis and antifungal activity of triazole and benzotriazole derivatives, European Journal of Medicinal Chemistry, 44 ,2009, 3064-3067.

[6]. V. Mathew, D. Giles, J. Keshavayya and V. Vaidya, Studies on synthesis and pharmacological activities of 1,2,4-triazolo[3,4-b]1,3,4thiadiazoles and their dihydro analogues, Arch Pharm (Weinheim), 342,2009, 210-22.

[7]. M.M.H. Bhuiyan1, M.I. Hossain,M.M. Mahmud ,Mohammad Al-Amin, , Microwave-assisted Efficient Synthesis of Chalcones as Probes for Antimicrobial Activities, Chemistry Journal,01,2011, 21-28 